\title{
Incidence and Prevalence of Inflammatory Bowel Disease in Norway and the Impact of Different Case Definitions: A Nationwide Registry Study
}

\author{
Sandre Svatun Lirhus (D) \\ Marte Lie Høivik ${ }^{2,3}$ \\ Bjørn Moum ${ }^{2,3}$ \\ Karoline Anisdahl ${ }^{2,3}$ \\ Hans Olav Melberg' \\ 'Department of Health Management and \\ Health Economics, University of Oslo, \\ Oslo, Norway; ${ }^{2}$ Department of \\ Gastroenterology, Oslo University \\ Hospital, Oslo, Norway; ${ }^{3}$ Institute of \\ Clinical Medicine, University of Oslo, \\ Oslo, Norway
}

Background: Countries have different diagnostic procedures and treatment regimens for inflammatory bowel disease (IBD) patients. In addition to differences in population characteristics, completeness of data and health registries, different follow-up time and case definitions can have a large impact on estimates of the incidence and prevalence of IBD.

Aim: The aim of this study was to use hospital and prescription data to estimate incidence and prevalence of Crohn's disease (CD) and ulcerative colitis (UC), using different case definitions.

Methods: This study used nationwide data from the Norwegian Patient Registry (2008 to 2017) and the Norwegian Prescription Database (2004 to April 2018). Incidence and prevalence were estimated using different case definitions of an IBD patient, varying the number of IBD-related hospital visits and IBD prescriptions required. The base case definition included patients with at least one IBD hospital visit and two IBD prescriptions or two IBD hospital visits.

Results: From 2010 to 2017, 16,758 incident IBD patients fulfilled our base case definition, with 6045 diagnosed with CD (36.1\%) and 10,713 (63.9\%) with UC. For CD, $47.2 \%$ of the patients were male while $53.8 \%$ of UC patients were male. The base case incidence varied between 14.1 and 16.0 per 100,000 person-years for CD and 24.7 and 28.4/100,000 personyears for UC patients in the years 2010-2017. When we required at least two IBD hospital visits, not utilizing the prescription data, the CD incidence was 22.3 per 100,000 personyears in 2010 and 13.9 per 100,000 person-years in 2017 . For UC, the incidence was 47.4 and 20.6 per 100,000 person-years in 2010 and 2017. In 2017, the prevalence of CD was $0.27 \%$ (95\% CI: $0.26-0.27)$ and $0.50 \%$ (95\% CI: $0.490-0.502)$ for UC.

Conclusion: According to our base case definition, the incidence of IBD in Norway was stable from 2010 to 2017. Both the incidence and prevalence of IBD in Norway is among the highest in the world. Moreover, the study also highlights the consequences of different case definitions.

Keywords: inflammatory bowel disease, incidence, prevalence, real-world data

\section{Introduction}

In Europe, the reported incidence of Crohn's disease (CD) ranges from 0.5 to 10.6 cases per 100,000 person-years and for ulcerative colitis (UC) the numbers are 0.9 to 24.3 per 100,000 person-years. ${ }^{1}$ The prevalence in Europe varies from 1.5 to 213 cases per 100,000 persons for CD and 2.4 to 294 cases per 100,000 persons for UC. ${ }^{1}$ The highest incidence and prevalence estimates have been reported in Northern Europe. The incidence of inflammatory bowel disease (IBD) has been
Correspondence: Sandre Svatun Lirhus Department of Health Management and Health Economics, University of Oslo, Forskningsveien 3A, Harald Schjelderups Hus, Oslo, 0373, Norway

Tel +4797582925

Email s.s.lirhus@medisin.uio.no 
reported to increase in the western world in the latter half of the 20th century. ${ }^{2,3}$ However, a recent systematic review of population-based studies from 1990 or later reported that $73 \%$ of $\mathrm{CD}$ and $83 \%$ of UC studies show stable or decreasing incidence in North America and Europe. ${ }^{4}$

The diagnosis of IBD is based on a combination of symptoms, endoscopy, radiology and histological findings in biopsies. ${ }^{5,6}$ Countries have different diagnostic procedures and treatment regimes, making comparisons of studies on the incidence and prevalence of IBD challenging. In addition to differences in population characteristics, completeness of data and health registries, different follow-up time and case definitions can have large impact for the estimates of IBD incidence and prevalence.

There are numerous studies that have estimated the incidence and prevalence of IBD using population-based registries. $^{1,4}$ Unlike Scandinavia, most countries do not have nationwide patient registries and data on IBD epidemiology is often derived from tertiary referral centers or insurance databases.

Potential problems related to limited follow-up and case definitions are addressed to a varying extent in the literature, but few studies have quantified the problem. Given this, the aim of this study was to use nationwide hospital and prescription data to estimate incidence and prevalence of IBD, using different case definitions.

\section{Materials and Methods}

\section{Data}

In Norway, all in- and outpatient hospital contacts are registered in the Norwegian Patient Registry (NPR), and it is mandatory to report diagnoses and clinical procedures. NPR dates back to 2008. The Norwegian Prescription Database (NorPD) includes information of all dispensed drug prescriptions by Norwegian pharmacies, including date (at pick-up time), and the Anatomical Therapeutic Chemical (ATC), International Classification of Diseases (ICD-10) and International Classification of Primary Carecodes (ICPC). Data from NorPD and NPR were linked using the unique personal identification number of every Norwegian resident, which made it possible to follow individual patients over time. The source population included all patients who received an IBD diagnosis (ICD-10 code K50 (CD) or K51 (UC)) at least once between 2008 and 2017 in NPR. Data from NorPD was from 2004 to 30 April 2018. Patients only registered with an IBD diagnosis in NorPD were not included as the data extraction was based on an IBD diagnosis in NPR.

\section{Definition of an IBD Prescription}

ICD- and ICPC-codes were not included in NorPD before March 2008 and were fully implemented in March 2009. ${ }^{7}$ For 5-aminosalicylic acid (5-ASA) prescriptions with a diagnosis code, 98.7\% had an IBD-code (ICD K50/51, ICPC D94). For budesonide prescriptions with a diagnosis code, 99.2\% had an IBD-code. These medications were therefore included as IBD prescriptions also when they were missing both an ICD- and ICPC-code in order to exclude prevalent patients prior to March 2008/2009. Other IBD-related prescriptions were only included if they had an IBD diagnosis code. Consequently, our definition of an IBD prescription included all pharmacy claims with ICD-code K50 or K51, ICPC-code D94 and/or prescriptions of 5-ASA or budesonide.

\section{Case Definitions}

Incidence and prevalence were estimated using different case definitions of an IBD patient, varying the number of IBD diagnosis required in NPR and IBD prescriptions from NorPD. For the incidence estimates including IBD prescriptions, patients with an IBD prescription more than 60 days prior to their first NPR registration were excluded. For prevalence, patients were defined as actively treated if they had at least one IBD visit in 2017 and at least one prescription for 5-ASA, corticosteroids, immunomodulators or biologic agents in $2017 .^{8}$

If a patient was registered with both $\mathrm{UC}$ and $\mathrm{CD}$, the last observed ICD-code in NPR determined whether the patient would be classified as CD or UC. Incident patients were included in the year of their first IBD hospital visit. Prevalent patients were included from the year of their first IBD visit or IBD prescription, whichever came first.

\section{Base Case Definition}

To avoid the inclusion of patients without IBD and a concurrent overestimation of incidence and prevalence, registry studies of IBD usually require two IBD-related hospital visits (ICD K50/51). ${ }^{8-11}$ When requiring two IBD visits, patients might be excluded if the follow-up time is too short to capture the second visit, and the problem increases the closer you get to the end of the study period. To deal with this fact, patients with only one IBD-related hospital event were accepted if there were two IBD prescriptions to compensate for only one hospital event. 
To distinguish incident and prevalent cases, a lookback period without an IBD diagnosis was required. Consequently, patients with their first IBD visit between 2008 and 2009 were excluded from the incidence estimates. Patients with an IBD prescription (in NorPD where we had available data going back to 2004) more than 60 days prior to their first NPR registration were excluded from the incidence estimates. See Figures S1 and $\underline{\mathrm{S} 2}$ to see the effect of varying the 60 -day window.

In the base case, incident IBD was defined as at least one IBD visit and two IBD prescriptions or two IBD visits. IBD visits included all in- and outpatient hospital visits with IBD.

\section{Statistical Analysis}

Statistics Norway (SSB) presents population statistics as the number of inhabitants on January 1st of every year. We calculated prevalence counting all incident and prevalent IBD patients alive on December 31st of each year, divided by inhabitants in Norway on January 1st of the succeeding year, presented as point-prevalence proportion with $95 \%$ CI. Incidence was estimated adding all incident patients, divided by the mid-year population in the same year, presented as incidence per 100,000 personyears with 95\% CI (exact Poisson confidence limits). The mid-year population was estimated taking the average of the population on January 1st of each year and January 1st of the succeeding year (eg the mean between 50-year-olds in 2011 and 51-year-olds in 2012).

Due to anonymization requirements from the health authorities, information on age was provided by birth cohort in 10-year intervals. Mean age is therefore not reported and age-specific incidence and prevalence was only calculated for the last available year (2017) since changes in age-specific incidence and prevalence over time could not be determined (Figures S3-S8). The respective age-intervals were chosen to ensure correct age groups. Data handling and analyses were performed using Python 3.X.

\section{Ethical Considerations}

The study was approved by the Norwegian Data Protection Authority and the Regional Committees for Medical and Health Research Ethics, NPR and NorPD.

\section{Results}

The source population included 51,488 IBD patients who had at least one IBD visit during $2008-2017$.

\section{Incidence}

From 2010 to 2017, 16,758 incident IBD patients fulfilled our base case definition, with 6045 diagnosed with CD (36.1\%) and 10,713 (63.9\%) with UC. Some of the incident patients were diagnosed with both $\mathrm{UC}$ and $\mathrm{CD}$ $(14.6 \%)$ in NPR and these were classified according to their last diagnosis. For $\mathrm{CD}, 47.2 \%$ of the patients were male while $53.8 \%$ of UC patients were male. The base case incidence varied between 14.1 and 16.0 per 100,000 person-years for CD and 24.7 and 28.4 per 100,000 person-years for UC patients in the years 2010 to 2017 (Figure 1).

When we required at least two IBD hospital visits, not excluding patients with an IBD prescription prior to their first IBD hospital visit, the CD incidence was 22.3 per 100,000 person-years in 2010 and 13.9 per 100,000 personyears in 2017. For UC, the incidence was 47.4 and 20.6 per 100,000 person-years in 2010 and 2017 (Figure 2). The incidence of IBD in Figure 2 was reduced when patients with at least two IBD visits were removed if they had received an IBD prescription more than 60 days prior to their first IBD visit (Figure 3).

When we required only one IBD visit, the incidence of $\mathrm{CD}$ increased from 14.6 (CI: 13.6-15.7) to 27.7 (CI: 24.3-27.1) per 100,000 person-years in 2017 (Figure 4). The incidence decreased to 13.9 (CI: 12.9-14.9) per 100,000 person-years when we required two IBD visits (Figure 4). With the strictest definition, requiring two IBD visits and one IBD prescription, the CD incidence was 10.1 (CI: 9.2-10.9) per 100,000 personyears. For UC, the base case incidence was 25.7 (CI: 24.3-27.1) per 100,000 person-years in 2017 (Figure 5). The incidence decreased to 20.6 (CI: 19.4-21.8) when we required two IBD visits. When we required two IBD visits and an IBD prescription, the incidence decreased to 16.4 (CI: 15.3-17.5).

The incidence of IBD for patients with a single IBD hospital visit and prescription can be seen in Figure S9. See Figure S10 for the incidence for patients with a single IBD hospital visit and no IBD prescriptions.

\section{Prevalence}

In 2017, 40,900 prevalent IBD patients fulfilled our base case definition, where 14,352 were diagnosed with CD (35.1\%) and 26,548 (64.9\%) with UC. For CD, 46.9\% of the patients were male, while $52.4 \%$ of UC patients were male. 


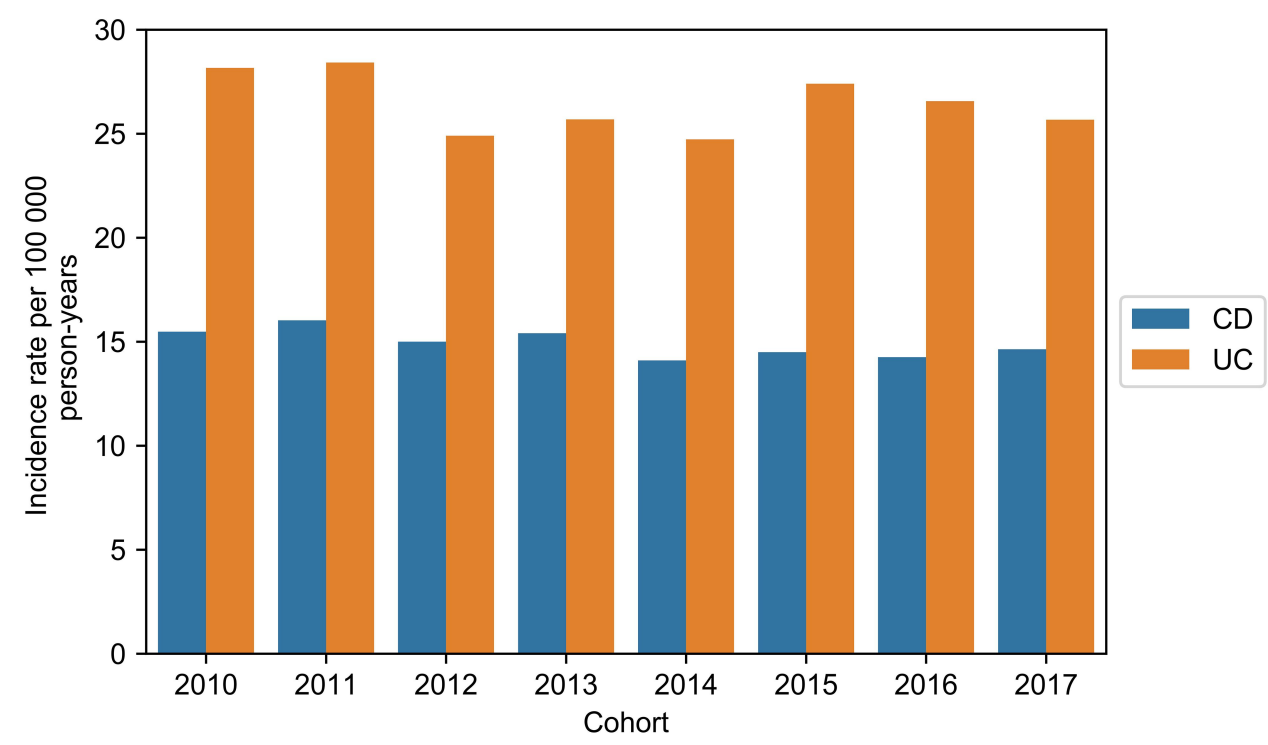

Figure I IBD incidence according to our base case definition: At least one IBD visit and two IBD prescriptions or two IBD visits. Patients were removed if they had received an IBD prescription more than 60 days prior to their first IBD visit.

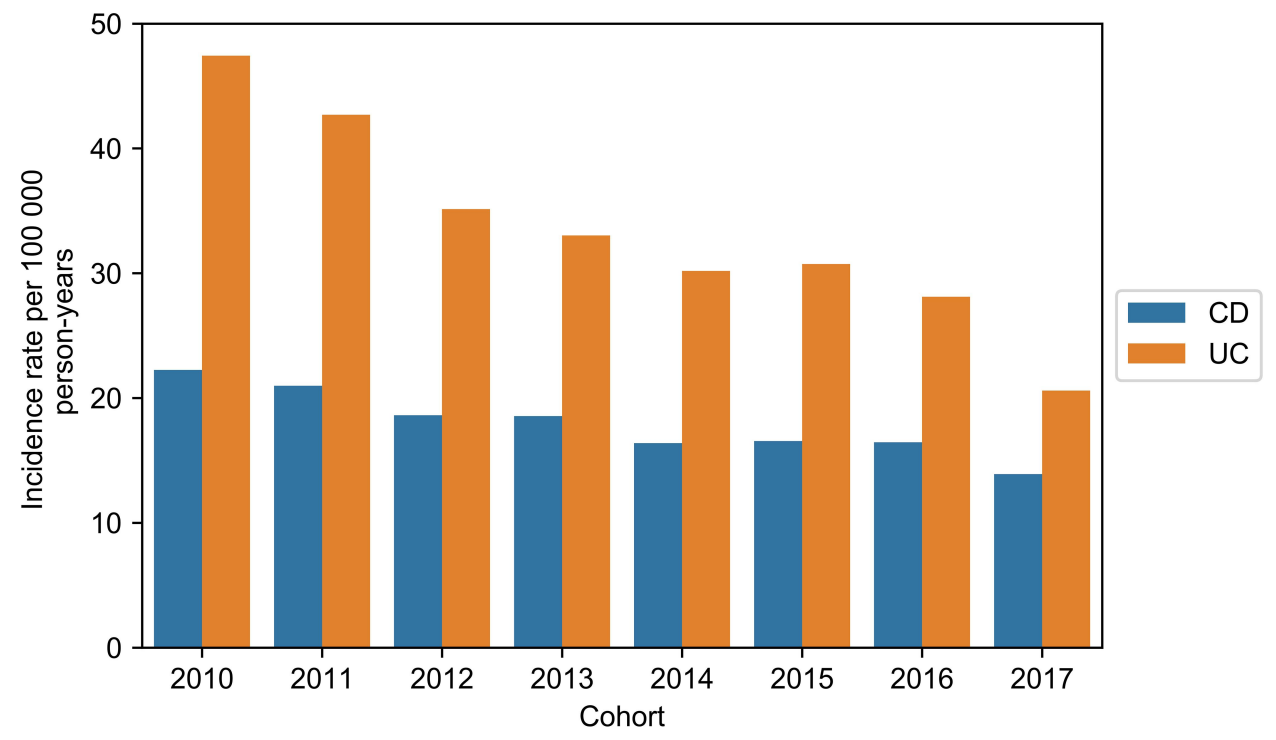

Figure 2 The incidence of IBD for patients with at least two records in the Norwegian Patient Registry (not utilizing prescription data).

The prevalence of CD was $0.27 \%$ (95\% CI: $0.26-0.27$ ) and $0.50 \%(95 \%$ CI: $0.49-0.50)$ for UC (Figures 6-8). When at least two IBD visits were required, the prevalence of CD decreased to $0.25 \%$ (95\% CI: $0.25-0.26$ ). For CD patients being actively treated (defined as one or more IBD visits in 2017 and at least one prescription of IBD-related drugs in 2017) the prevalence was $0.15 \%$ (95\% CI: $0.15-$ $0.15)$. For UC, the prevalence decreased to $0.42 \%(95 \%$ CI: $0.42-0.43$ ) when two IBD visits were required and for patients being actively treated the prevalence was $0.21 \%$ (95\% CI: $0.21-0.22$ ).

\section{Discussion}

This study provides the first Norwegian estimate of incidence and prevalence of IBD based on nationwide data. According to this study, both incidence and prevalence of IBD in Norway is among the highest in the world. In 2017, the incidence of IBD was 40 (CD: 14.6, UC: 25.7) per 100,000 person-years and the prevalence was $0.77 \%$ (CD: $0.27 \%$, UC: $0.50 \%$ ). The base case definition resulted in higher incidence and prevalence estimates in 2017 compared to the more commonly used definition of at least two hospital visits. 


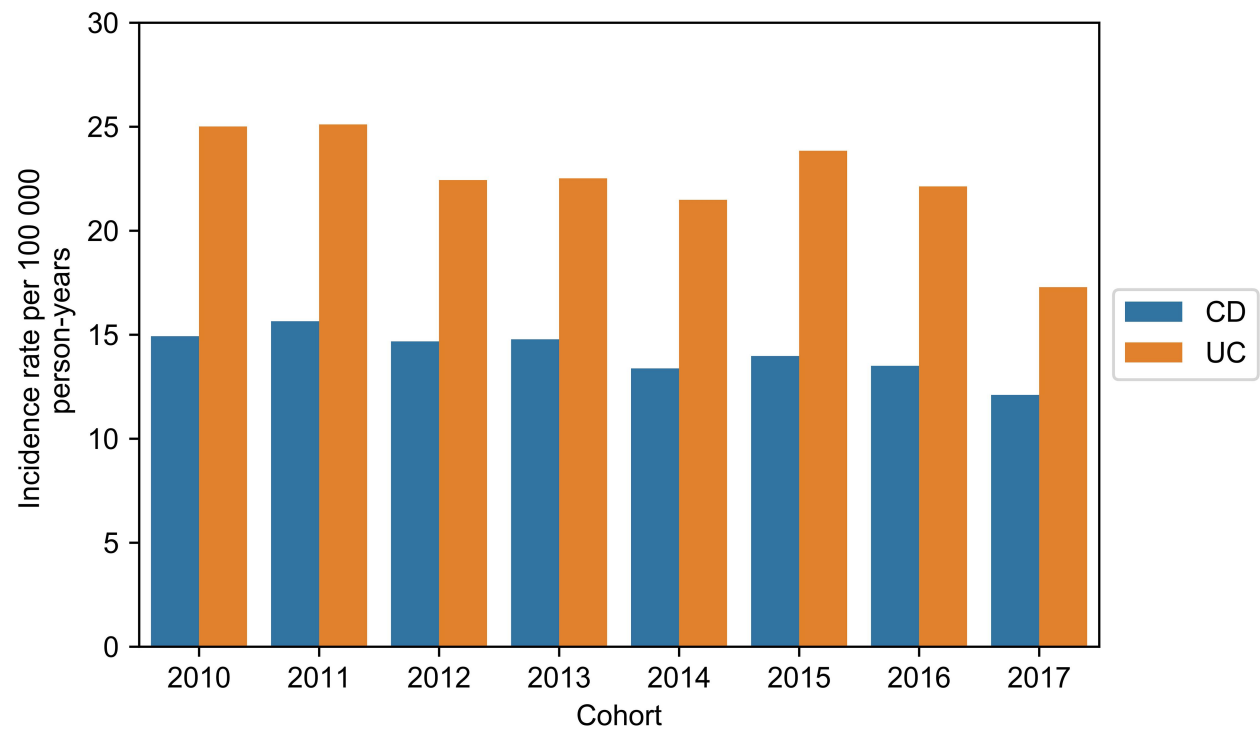

Figure 3 The incidence of IBD for patients with at least two records in the Norwegian Patient Registry. Patients were removed if they had received an IBD prescription more than 60 days prior to their first IBD visit.

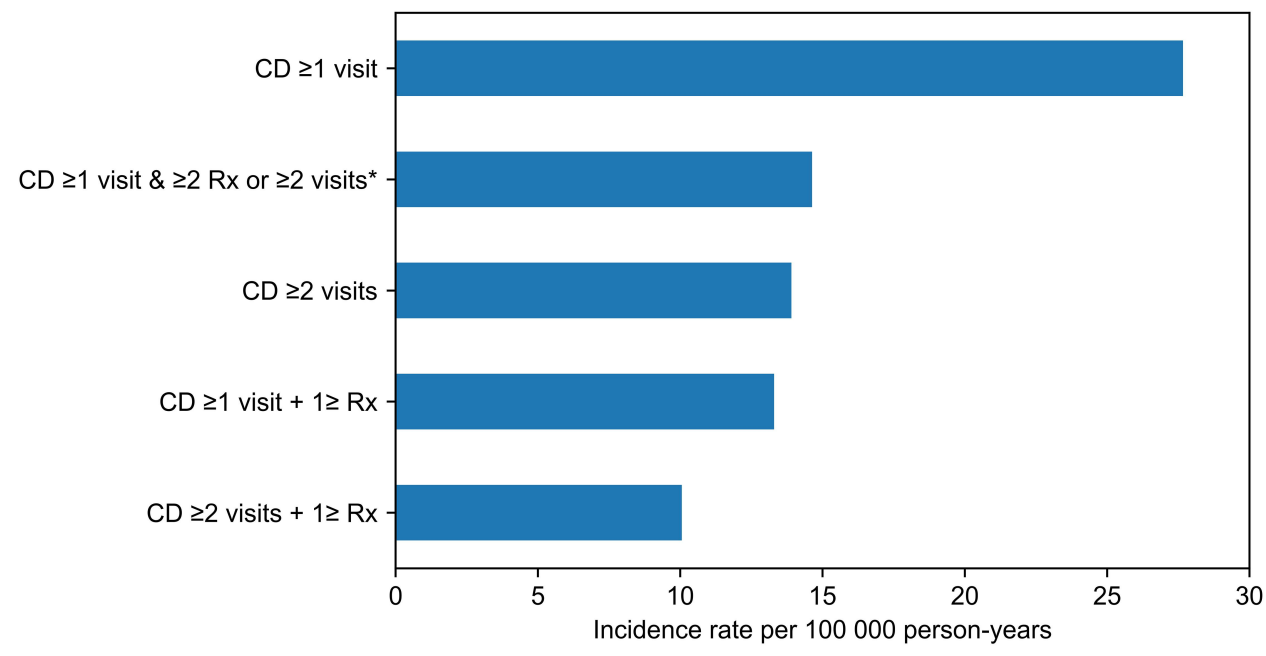

Figure $4 \mathrm{CD}$ incidence in 2017 according to different case definitions. *Base case definition.

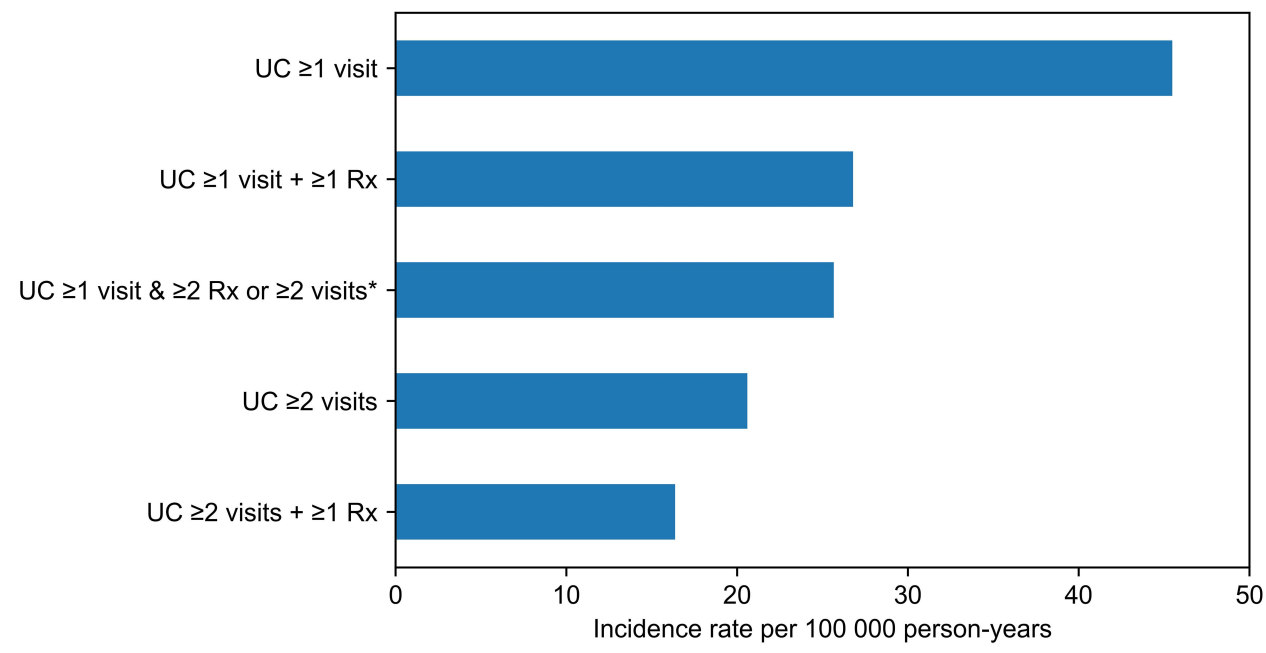

Figure 5 UC incidence in 2017 according to different case definitions. *Base case definition. 


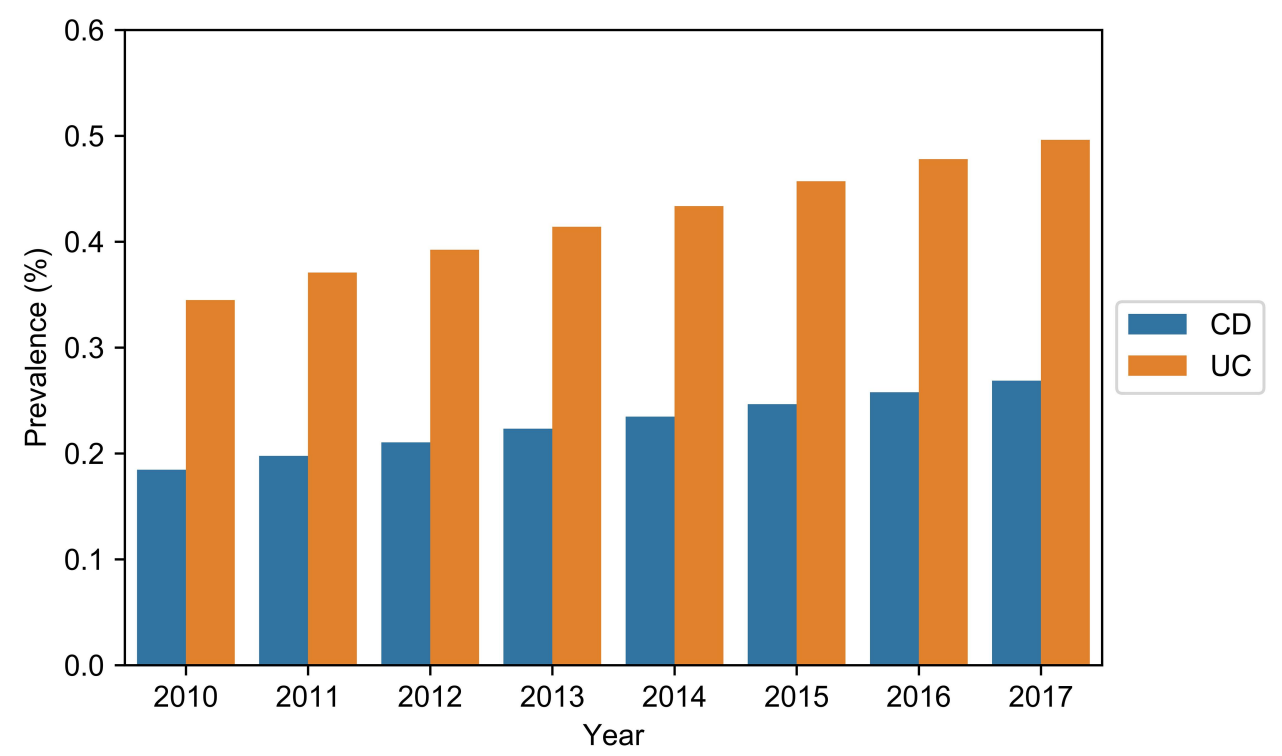

Figure 6 IBD prevalence according to our base case definition: At least one IBD visit and two IBD prescriptions or two IBD visits.

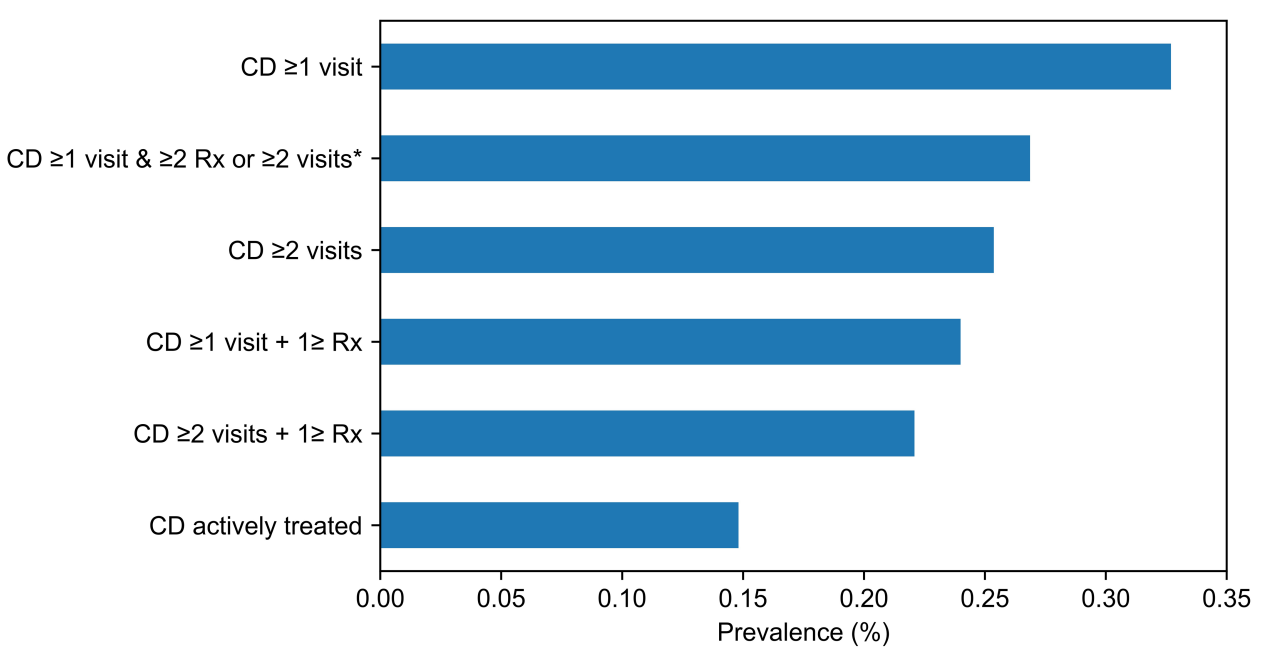

Figure 7 CD prevalence in 2017 according to different case definitions. Actively treated was defined as one or more IBD visits in 20 I7 and at least one prescription of IBDrelated drugs in 2017. *Base case definition.

\section{Prevalence}

The prevalence of CD and UC increased linearly from 2010 to 2017. However, we cannot conclude that there is an increasing prevalence in Norway due to the relatively short data inclusion period. Previous Norwegian estimates from 2009 were $0.26 \%$ for CD and $0.51 \%$ for UC. ${ }^{12}$ Despite the use of a different methodology, the estimate from 2009 is very close to this study's base case estimation for 2017.

A Swedish study using National Patient Registry data from 1987 to 2010 reported an IBD prevalence of $0.65 \%$ (CD: $0.35 \%$, UC: $0.19 \%$ and IBDU: $0.11 \%$ ) in 2010 requiring two IBD visits. ${ }^{8}$

\section{Incidence}

A population-based prospective study from the early nineties including one-fourth of Norway's population estimated the incidence of IBD to 19.3 (CD: 5.1, UC: 10.6 and IBDU: 3.6) per 100,000 population. ${ }^{13}$ This would suggest an increasing incidence of IBD over the last 25 years in Norway. However, there was no clear trend in incidence over the eight years in our study. An increase could have been masked by an underestimation of the incidence in the later years of the study due to the case definition requirement of a second IBD visit and/or two IBD prescriptions. 


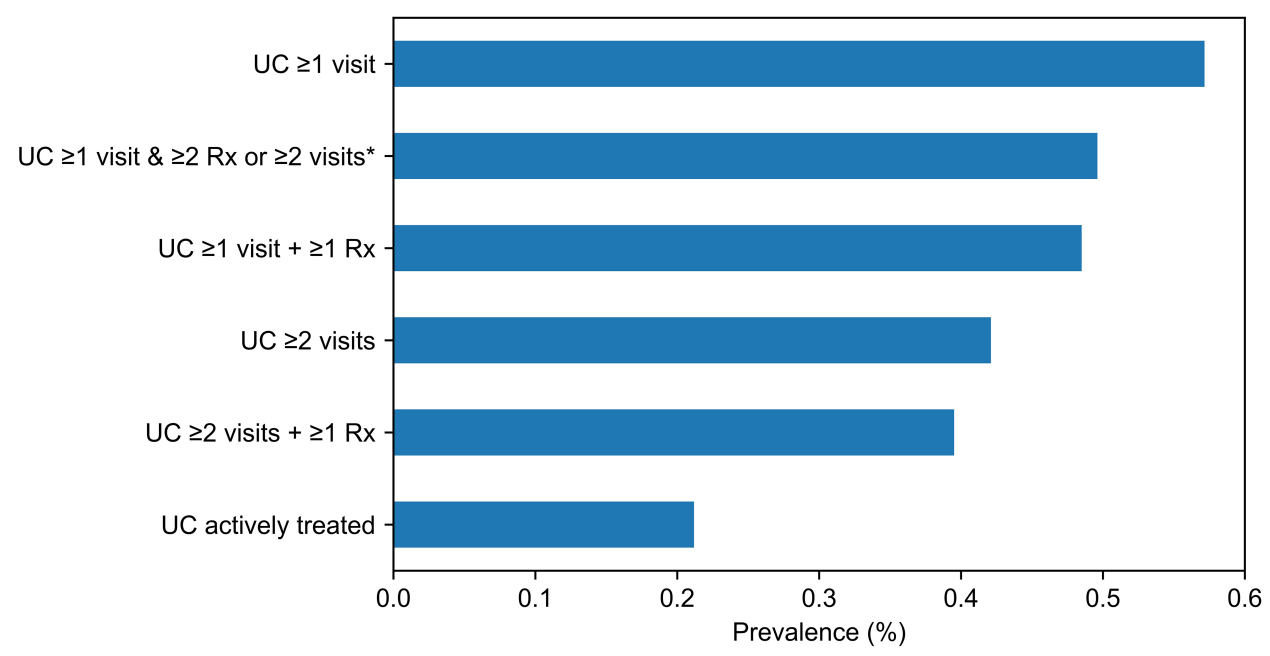

Figure 8 UC prevalence in 2017 according to different case definitions. Actively treated was defined as one or more IBD visits in 2017 and at least one prescription of IBDrelated drugs in 2017 . *Base case definition.

A Danish study using nationwide registry data from 1977 to 2013 reported an incidence of 9.1 per 100,000 person-years for CD and 18.6 per 100,000 for UC in the period of 2010-13. ${ }^{9}$ However, when breaking down the incidence per year, there was a substantial decrease in the incidence from 2010 to 2011 (CD: 11.6, UC: 26.3) to 2012-13 (CD: 6.6, UC: 10.7) since patients were required to have two IBD visits (visually estimated from Figure 1 of Lophaven et al. ${ }^{9}$ ). The incidence estimates from 2010 to 2011 suggest that the IBD incidence is comparable between Norway and Denmark. The reported median time between the first two recordings in the Danish study was 430 days (CD) and 654 days (UC) which emphasizes the probability of an underestimation of incidence in the later years of a registry study.

The optimal case definition depends on the data period, registration practice and health care system. A Canadian study tested more than 5000 algorithms and found the most accurate case definition to be five physician contacts or hospitalizations within 4 years (for patients 18-64 years at diagnosis). ${ }^{14}$ If we would have used this definition, we would not have been able to calculate the incidence for the last years of our study period. It also introduces problems with the date of diagnosis for patients who have a GP visit with an IBD diagnosis prior to their first hospital registration with IBD. A Swedish study reported a positive predictive value of $93 \%$ for any IBD requiring two IBD hospital visits. ${ }^{11}$ The criteria of at least two hospital visits would lead to an overestimation in the earlier years due to the short lookback period and underestimation in the later years. The overestimation in earlier years is apparent in
Figure 2, demonstrating the value of including prescription data to classify incident patients. In addition to reducing the misclassification of prevalent cases as incident cases, the difference between Figures 2 and 3 suggests that patients get treatment outside specialist healthcare for longer periods as they receive IBD prescriptions without having hospital contacts.

The main strength of the study is the use of a complete and representative dataset for the whole population across many years, for both hospital events and prescription events. This means that the results are not subject to statistical sample uncertainty.

A limitation of the study is the handling of patients registered with both CD and UC which includes $14.6 \%$ of the base case incidence. We did not included them as a third separate IBD unclassified (IBD-U) group since that would remove nearly three times more patients in 2010 compared to 2017. This is because the probability of receiving both an UC and $\mathrm{CD}$ diagnosis increases with longer follow-up time. This indicates that there are multiple reasons for a patient to be registered with both UC and CD: IBD-U, wrong ICD-code registered due to human error, misdiagnosis or a change from $\mathrm{UC}$ to $\mathrm{CD}$. Since the proportion of incident patients who are registered with $\mathrm{UC}$ and $\mathrm{CD}$ varies over time, we did not want to claim or give the impression that this was one coherent group (IBD-U) by including it as a unified category. Excluding the group would also be a mistake, since this would underestimate the incidence. We acknowledge that there is no perfect way to solve this issue with patients registered with both $\mathrm{UC}$ and $\mathrm{CD}$. 
In summary, our base case definition tried to include all incident IBD patients and avoid classifying prevalent patients as incident cases. It is difficult to assess the accuracy of the definition, but we believe that our base case definition is a step forward compared to the other estimations discussed in this study.

\section{Conclusion}

According to our base case definition, the incidence of IBD in Norway was stable from 2010 to 2017. Both the incidence and prevalence of IBD in Norway is among the highest in the world. Moreover, the study also highlights the consequences of different case definitions.

\section{Funding}

This work was financially supported by Takeda.

\section{Disclosure}

SSL reports research funding from Takeda during the conduct of the study and personal fees from Takeda outside the submitted work. HOM reports research funding from Takeda during the conduct of the study and personal fees from Takeda outside the submitted work. MLH reports unrestricted research grants from Tillotts, Ferring and Takeda; advisory board for Takeda; and speaker fees from AbbVie, Meda and Takeda. BM serves in the advisory board for AbbVie, Jansen, Hospira, Orion Pharma, Pfizer, Sandoz, Vifor Pharma; reports speaker fees from Takeda, Pfizer, Orion Pharma, Hospira, Ferring, and Cosmo Pharma. KA reports personal fees from Takeda. The authors report no other conflicts of interest in this work.

\section{References}

1. Burisch J, Jess T, Martinato M, Lakatos PL. The burden of inflammatory bowel disease in Europe. J Crohns Colitis. 2013;7(4):322-337. doi:10.1016/j.crohns.2013.01.010

2. Molodecky NA, Soon IS, Rabi DM, et al. Increasing incidence and prevalence of the inflammatory bowel diseases with time, based on systematic review. Gastroenterology. 2012;142(1):46-54.e42. doi:10.1053/j.gastro.2011.10.001

Clinical Epidemiology

\section{Publish your work in this journal}

Clinical Epidemiology is an international, peer-reviewed, open access, online journal focusing on disease and drug epidemiology, identification of risk factors and screening procedures to develop optimal preventative initiatives and programs. Specific topics include: diagnosis, prognosis, treatment, screening, prevention, risk factor modification,

Submit your manuscript here: https://www.dovepress.com/clinical-epidemiology-journal
3. Kaplan GG, Ng SC. Understanding and preventing the global increase of inflammatory bowel disease. Gastroenterology. 2017;152(2):313-321.e2. doi:10.1053/j.gastro.2016.10.020

4. Ng SC, Shi HY, Hamidi N, et al. Worldwide incidence and prevalence of inflammatory bowel disease in the 21 st century: a systematic review of population-based studies. Lancet. 2017;390 (10114):2769-2778. doi:10.1016/S0140-6736(17)32448-0

5. Henriksen M, Jahnsen J, Lygren I, et al. Change of diagnosis during the first five years after onset of inflammatory bowel disease: results of a prospective follow-up study (the IBSEN Study). Scand $J$ Gastroenterol. 2006;41(9):1037-1043. doi:10.1080/003655206 00554527

6. Van Assche G, Dignass A, Panes J, et al. The second European evidencebased Consensus on the diagnosis and management of Crohn's disease: definitions and diagnosis. Elsevier Enhanced Reader. Available from: https://reader.elsevier.com/reader/sd/pii/S1873994609001469?token= B0C6C12C79343D3D5603FCBEB44707A86E24DAFB0A5F2A056E E7984ED051BD5606143C94402E03A01FBEFD7009D96DAF. Accessed February 20, 2020.

7. Norwegian Institute of Public Health. The Norwegian Prescription Database 2012-2016; 2017. Available from: https:/www.fhi.no/con tentassets/10528e 05afc141408bc8a8fb5dfb7109/reseptregisteret2012-2016.pdf. Accessed April 12, 2021.

8. Büsch K, Ludvigsson JF, Ekström-Smedby K, Ekbom A, Askling J, Neovius M. Nationwide prevalence of inflammatory bowel disease in Sweden: a population-based register study. Aliment Pharmacol Ther. 2014;39(1):57-68. doi:10.1111/apt.12528

9. Lophaven SN, Lynge E, Burisch J. The incidence of inflammatory bowel disease in Denmark 1980-2013: a nationwide cohort study. Aliment Pharmacol Ther. 2017;45(7):961-972. doi:10.1111/ apt. 13971

10. Everhov ÅH, Halfvarson J, Myrelid P, et al. Incidence and treatment of patients diagnosed with inflammatory bowel diseases at 60 years or older in Sweden. Gastroenterology. 2018;154(3):518-528.e15. doi:10.1053/j.gastro.2017.10.034

11. Jakobsson GL, Sternegård E, Olén O, et al. Validating inflammatory bowel disease (IBD) in the Swedish National Patient Register and the Swedish Quality Register for IBD (SWIBREG). Scand $J$ Gastroenterol. 2017;52(2):216-221. doi:10.1080/00365521.20 16.1246605

12. Bengtson M-B, Solberg C, Aamodt G, et al. Familial aggregation in Crohn's disease and ulcerative colitis in a Norwegian populationbased cohort followed for ten years. J Crohns Colitis. 2009;3 (2):92-99. doi:10.1016/j.crohns.2008.11.002

13. Moum B, Vain MH, Ekbom A, et al. Incidence of inflammatory bowel disease in Southeastern Norway: evaluation of methods after 1 year of registration. DIG. 1995;56(5):377-381. doi:10.1159/ 000201262

14. Benchimol EI, Guttmann A, Mack DR, et al. Validation of international algorithms to identify adults with inflammatory bowel disease in health administrative data from Ontario, Canada. J Clin Epidemiol. 2014;67(8):887-896. doi:10.1016/j.jclinepi.2014.02.019 systematic reviews, risk \& safety of medical interventions, epidemiology \& biostatistical methods, and evaluation of guidelines, translational medicine, health policies \& economic evaluations. The manuscript management system is completely online and includes a very quick and fair peer-review system, which is all easy to use. 